\title{
Correction to: Application of chain transfer agents in the two-part dispersion polymerization of cross-linked polymer microspheres
}

\author{
Binbin $\mathrm{Lv}^{1} \cdot$ Hui Wang ${ }^{2,3} \cdot$ Liang $\mathrm{He}^{2} \cdot$ Xueyan $\mathrm{Nan}^{2} \cdot$ Tong Wang $^{2} \cdot$ Zhizhou Liu $^{2} \cdot$ Wei Xia ${ }^{1} \cdot$ Pengli Bai ${ }^{2,4}$
}

Published online: 18 August 2021

○ Springer-Verlag GmbH Germany, part of Springer Nature 2021

\section{Colloid and polymer science \\ https://doi.org/10.1007/s00396-021-04843-4}

In this article the correction in affiliation assignment were incorrectly carried out. The authors list should be Binbin $\mathrm{Lv}^{1}$, Hui Wang ${ }^{2,3}$, Liang $\mathrm{He}^{2}$, Xueyan $\mathrm{Nan}^{2}$, Tong Wang ${ }^{2}$, Zhizhou $\mathrm{Liu}^{2}$, Wei Xia ${ }^{1}$, Pengli Bai ${ }^{2,4}$.

The original article has been corrected.

Publisher's Note Springer Nature remains neutral with regard to jurisdictional claims in published maps and institutional affiliations.

The original article can be found online at https://doi.org/10.1007/ s00396-021-04843-4.

Wei Xia

496966717@qq.com

1 Beihua University, Jilin 132013, People's Republic of China

2 CAS Key Lab of Bio-Medical Diagnostics, Suzhou Institute of Biomedical Engineering and Technology,

Chinese Academy of Sciences, Suzhou, Jiangsu 215163,

People's Republic of China

3 School of Life Sciences, Shanghai University, Shanghai 200444, People's Republic of China

4 Jihua Laboratory, Nanhai District, No.28 Island Ring South Road, Guicheng StreetFoshan City, Guangdong Province,

China 